\section{O sistema de informações sobre mortalidade: problemas e propostas para o seu enfrentamento \\ II - Mortes por causas externas}

\section{The national mortality information system: problems and proposals for solving them II - Deaths due to external causes}

\author{
Maria Helena Prado de Mello Jorge \\ Departamento de Epidemiologia \\ Faculdade de Saúde Pública \\ Universidade de São Paulo \\ Correspondência para/Correspondence to: \\ Av. Dr. Arnaldo, 715 \\ 01246-904 - São Paulo, SP, Brasil \\ mhpjorge@usp.br
}

\section{Sabina Léa Davidson Gotlieb}

Departamento de Epidemiologia

Faculdade de Saúde Pública

Universidade de São Paulo

\section{Ruy Laurenti}

Departamento de Epidemiologia

Faculdade de Saúde Pública

Universidade de São Paulo

\section{Auxílio financeiro}

Fundação de Amparo à Pesquisa do Estado de São Paulo - FAPESP (Processo No 98-15272-4) e Conselho Nacional de Desenvolvimento Científico e Tecnológico - CNPq (Processo No 520311-96-5)

\section{Resumo}

As causas externas representam importante fator na mortalidade do Brasil. Conhecer quem é vulnerável e quais os motivos disso constituem um elemento fundamental para políticas de prevenção de acidentes e violências. Para que esses programas sejam efetivos, é necessário um detalhamento sobre as reais causas de morte. $\mathrm{O}$ objetivo desta pesquisa foi verificar, dentre as declarações de óbito (DO) por causas externas, aquelas que poderiam ter causas básicas melhor descritas, bem como o ganho possível diante de metodologia do resgate/recuperação da informação. Foram estudados 320 óbitos do último trimestre de 2000, de quinze cidades com características demográficas e socioeconômicas heterogêneas. A metodologia baseou-se em visitas aos Institutos de Medicina Legal e Delegacias de Polícia (com consulta aos laudos e Boletins de Ocorrência) e aos domicílios dos falecidos. Do total, $164(51,3 \%)$ já poderiam ser considerados bem definidos, em uma primeira análise; dos demais, 83 constituíam óbitos por causa externa do tipo ignorado e 73, diagnóstico incompleto de causa externa. Após a investigação houve mudanças apreciáveis na distribuição dessas mortes: o total de homicídios foi cinco vezes maior; os suicídios dobraram sua freqüência; e os acidentes de transporte tornaram-se $90 \%$ a mais do que os informados pelas DO originais, diminuindo sensivelmente as mortes por causas externas de tipo ignorado. Embora se reconheça que as mortes por violências e acidentes sejam melhor informadas do que as naturais, há ainda muito a ser feito enquanto os legistas não alcançarem um patamar ideal no preenchimento das DO. A adoção desta metodologia, simples, acessível e exeqüível, possibilita o desejado aprimoramento das estatísticas de mortalidade.

Palavras-chave: Causas externas. Mortalidade. Qualidade da informação. 


\section{Abstract}

Given that the importance of morbidity and mortality due to accidents and violence is increasing in Brazil and that, coroners frequently do not fill out the death certificate (DC) with the external cause which prompted the lesion that led to death, a decision was made to analyze all these deaths, even cases in which the information in the original DC could be considered appropriate. The aim of this research was to verify the level and amount of proper information put into the DC and up to what extent the ones labeled as inadequate could be enhanced; it also aimed to verify the gains provided by using a very simple method for recovering information. There were 320 deaths due to external causes, in the last quarter of 2000, in 15 Brazilian municipalities with heterogeneous demographic, social and economic characteristics, in the states of Sergipe, São Paulo, Mato Grosso. The clarification of each situation was obtained through consultation to autopsy reports in Forensic Institutes, reports at police stations and, whenever necessary, home visits. The results showed important changes in these causes of death. The numbers of deaths due to homicides, suicides and transportation accidents grew, respectively, six fold, two-fold and $90 \%$ after the investigation in relation to the original DC; and there was a significant decrease in deaths due to unknown external causes. Although deaths due to external causes are known to be better informed than deaths due to natural causes, there is much to be done in order to solve the problem of ill-defined causes. The adoption of this very simple and accessible methodology can lead to the desired improvement in these data.

Keywords: Mortality. External causes. Quality of information.

\section{Introdução}

A importância dos acidentes e violências como causa de morte vem crescendo a cada dia, situação que tem sido mostrada por diversos pesquisadores ${ }^{1-4}$. Sua freqüência mostra-se elevada e a ocorrência em faixas etárias baixas, principalmente de adolescentes e de jovens, empresta ao Brasil o título de quase campeão em relação ao indicador de nível de saúde Anos Potenciais de Vida Perdidos. Estudo recente mostrou que, para o Estado de São Paulo, a eliminação da violência como causa de morte representaria ganho de pelo menos dois anos na esperança de vida, sendo que na Região Metropolitana seu valor alcançaria 4 anos $^{5}$.

As informações sobre esses eventos constituem elementos importantes para o monitoramento de sua tendência no país e, conseqüentemente, do impacto de intervenções e políticas públicas voltadas para a sua redução. Assim, conhecer quem é vulnerável, em que grau e por que motivos, é imperioso quando se pensa em termos de prevenção e, nesse sentido, a informação completa, correta e fidedigna, principalmente quanto à causa da morte, mostra-se imprescindível.

A análise das mortes por causas externas pressupõe, a priori, o esclarecimento de alguns pontos. A Organização Mundial de Saúde - OMS - informa que, sempre que a causa da morte for uma lesão ou outro efeito de uma causa externa classificada no capítulo XIX da Classificação Internacional de Doenças - 10ª Revisão (CID-10), as circunstâncias que deram origem a essa afecção deverão ser selecionadas como causa básica para tabulação e codificadas entre V01 e Y896. Esse princípio deixa claro que as causas básicas corresponderão aos tipos de causas externas (englobadas no capítulo XX da CID10) que originaram as lesões e nunca a essas lesões. A lógica desta orientação é a de que, do ponto de vista da prevenção, não adianta conhecer a natureza da lesão (por exemplo, traumatismo, ferimento, hemorragia); é necessário precisar os tipos de agravos (atropelamento por ônibus, homicídio por arma 
de fogo, queda acidental de janela etc.) que ocasionaram essas lesões para, assim, poder preveni-las.

Outro ponto a ser levantado é o de que a lei brasileira determina que, nas mortes suspeitas ou decorrentes de causas externas (não conseqüentes a doenças), a Declaração de Óbito (DO) seja fornecida sempre por perito legista após necropsia (Código de Processo Penal, art. 262). Os médicos legistas, entretanto, não poucas vezes colocam no atestado somente a chamada "causa médica” da morte (natureza da lesão), negligenciando o aspecto relativo ao tipo de causa externa. Este fato levou o Ministério da Saúde a incluir na DO, a partir da segunda metade da década de 90, as variáveis do campo VIII (Prováveis circunstâncias da morte não natural), estas também, entretanto, nem sempre preenchidas. Trabalho de Laurenti et al. ${ }^{7}$, feito a partir de 6.279 declarações de óbito do Estado do Rio de Janeiro, em 1999, mostrou que, especificamente para a variável 59 - "Descrição sumária do evento", o que fora anotado pelo médico possibilitou completa descrição das circunstâncias do acidente/violência em $56,4 \%$ dos casos; em $30,9 \%$ o campo estava em branco, aparecendo os demais $12,7 \%$ anotados de forma a não permitir a identificação da real causa básica da morte, já que, na grande maioria dos casos, a informação prestada correspondia ao que já fora descrito nas Partes I e II da DO.

A ausência da informação adequada nas DO tem levado alguns Serviços de Estatística/Epidemiologia das Secretarias de Saúde/ Planejamento, responsáveis pela codificação das causas de morte, a procurar os Institutos de Medicina Legal visando ao aprimoramento das causas declaradas, já que, desde 1979, alguns autores têm afirmado que estas instituições dispõem de dados minuciosos, apenas não os transcrevendo para as $\mathrm{DO}^{8,9}$. A total ausência de informação, apesar disso, continua a existir, sendo que no Brasil a porcentagem de mortes por causas externas em que não se sabe sequer se decorrentes de homicídio, suicídio ou acidente, foi estimada ao redor de $10 \%$ no final da década de $90^{2}$, embora em época não muito distante chegasse a mais de $50 \%$ em alguns municípios brasileiros, como no Rio de Janeiro ${ }^{10}$. É importante salientar que percentuais elevados de causas externas de tipo ignorado constituem um obstáculo para o estabelecimento de um real panorama epidemiológico.

Este trabalho visa analisar as mortes por acidentes e violências, na perspectiva de avaliação do SIM/MS, já referida em outro trabalho ${ }^{11}$. O objetivo da análise dos óbitos por causas externas foi verificar o nível de preenchimento das DO e em que medida a informação poderia ser melhorada. Visou ainda conhecer, em todos os casos em que houve mudança de causa básica, qual a nova causa considerada. Este processo permite estabelecer uma verdadeira rede de "migração" de causas desconhecidas (ou mal informadas) para outras, analisando possíveis ganhos quanto ao detalhamento das causas e a conseqüente modificação do quadro epidemiológico.

\section{Material e Métodos}

Tendo em vista que, muitas vezes, os legistas não colocam na DO o tipo de causa que provocou a lesão que originou a mor$\mathrm{te}^{12,13}$, optou-se por trabalhar com este grupo, analisando inclusive os casos em que a informação constante na DO original já poderia ser considerada de certa forma adequada, do ponto de vista do correto preenchimento da causa básica da morte.

Foram selecionados óbitos por causas externas (capítulo XX da CID-10) ${ }^{6}$ ocorridos no último trimestre de 2000 nos quinze municípios referidos em trabalho anterior ${ }^{11}$, sendo três em Sergipe, cinco em São Paulo e sete em Mato Grosso.

$\mathrm{O}$ resgate das informações sobre cada morte se deu por meio de consultas ao laudo necroscópico no Instituto Médico Legal e ao Boletim de Ocorrência Policial nas Delegacias de Polícia ou em visita domiciliar. Essas informações foram anotadas em formulários especialmente preparados para esse fim. 
O total de casos analisados foi igual a 320 óbitos por acidentes e violências, sendo 164 bem definidos e 156 não definidos. Alguns foram alocados nas categorias de causas externas de tipo totalmente não definido (Y10 a Y34 da CID-10), casos em que era possível detectar somente tratar-se de morte decorrente de causa externa.

Outros casos foram considerados como diagnósticos incompletos nas causas externas, tendo sido assim distribuídos:

- acidentes de transporte não especificados (X89) = 35 óbitos

- demais acidentes $=31$ óbitos

quedas não especificadas $($ W19) $=2$ óbitos

afogamentos não especificados $(\mathrm{W} 74)=13$ óbitos

acidentes com fogo ou chamas não especificados (X09) = 2 óbitos

exposição acidental a fatores não especificados $(\mathrm{X} 59)=14$ óbitos

- $\quad$ suicídios não especificados $(\mathrm{X} 84)=2$ óbitos

- homicídios não especificados (Y09) = 5 óbitos.

Esses casos foram chamados diagnósticos incompletos de nível I.

Finalmente, dentre os considerados bem definidos para uma macro-análise, selecionaram-se alguns que ainda poderiam ter a causa melhor especificada (casos denominados “diagnósticos incompletos de nível II”).

\section{Resultados e Discussão}

Nos quinze municípios estudados ocor- reram, no período, 320 óbitos por causas externas. Destes, 164, correspondendo a $51,3 \%$, para uma primeira análise, foram considerados como bem definidos, sobressaindo o fato de que $88,2 \%$ dos suicídios e $92,8 \%$ dos homicídios encontravam-se nessa condição. Quanto às mortes acidentais verificou-se que $65 \%$ dos acidentes de transporte e $39,2 \%$ dos demais acidentes apresentaram-se adequadamente informados (Tabela 1).

Os demais 156 casos distribuíram-se em 83 óbitos, para os quais se sabia apenas tratar-se de morte decorrente de acidente ou violência (Y10 a Y34 da CID-10), razão pela qual foram designados como "causas externas mal definidas”, e 73 óbitos foram considerados como decorrentes de "diagnósticos incompletos de causas externas”.

\section{Óbitos por causas externas mal definidas}

Este grupo de causas foi eqüivalente aos casos mal definidos, pois nada se sabia sobre o tipo de causa externa que levara à morte, a não ser tratar-se de óbito decorrente de uma lesão e não de causa natural; estiveram nessa situação 83 casos. Em 54 casos a informação era totalmente ignorada (Y34), pois em geral o que estava anotado na DO era somente a natureza da lesão: traumatismo, hemorragia, ferimento etc.; em 29 eventos foi possível saber, pelo menos, que 15 mortes haviam sido provocadas por arma de fogo, sete por instrumento cortante, cinco por instrumento contundente, uma por sufocação e uma por outro meio espe-

Tabela 1 - Número e proporção (\%) de óbitos por causas externas segundo tipo e motivo de análise. Projeto SIM; 2000. Table 1 - Number and proportion (\%) of deaths due to external causes according to type and reason of analysis. Project SIM; 2000

\begin{tabular}{lcccccccc}
\hline TIPOS & \multicolumn{2}{c}{ BEM DEFINIDO } & \multicolumn{2}{c}{ MAL DEFINIDO } & \multicolumn{2}{c}{ DIAGNÓSTICOS } & \multicolumn{2}{c}{ TOTAL } \\
& & & & & & & \multicolumn{2}{c}{ INCOMPLETOS } \\
& No & $\%$ & No & $\%$ & No & $\%$ & No & $\%$ \\
\hline Ac. transporte & $65^{*}$ & 65,0 & - & - & 35 & 35,0 & 100 & 100,0 \\
Demais acidentes & 20 & 39,2 & - & - & 31 & 60,8 & 51 & 100,0 \\
Suicídios & 15 & 88,2 & - & - & 2 & 11,8 & 17 & 100,0 \\
Homicídios & 64 & 92,8 & - & - & 5 & 7,2 & 69 & 100,0 \\
Ignorados & - & - & 83 & 100,0 & - & - & 83 & 100,0 \\
TOTAL & 164 & 51,3 & 83 & 25,9 & 73 & 22,8 & 320 & 100,0 \\
\hline
\end{tabular}

" 44 considerados como diagnósticos incompletos nível II / 44 considered as incomplete diagnosis level II 
cificado, sem se conhecer, entretanto, a intencionalidade dessas mortes, dado o fato de não existir na DO original menção que levasse a distinguir tratar-se de acidente, suicídio ou homicídio (Tabela 2).

Após a investigação verificou-se terem ocorrido 15 perdas $(18,1 \%)$ e, dos casos restantes, foi possível definir melhor 56 casos $(82,3 \%)$, permanecendo na categoria "ignorado” apenas $12(17,7 \%)$ (proporções calculadas sobre o total de casos com informação conhecida). A Tabela 2 mostra, para o conjunto dos municípios, para que tipo de causa houve migração.

Em dois casos (2,9\%) a nova causa básica, segundo regras de codificação da CID10, foi considerada natural: o primeiro era queda levando à fratura e pôde-se constatar que a paciente era portadora de osteoporose, tendo a fratura sido decorrente desta patologia; o segundo caso era uma queda conseqüente a ataque epiléptico, sendo, portanto, a epilepsia a causa básica da morte.

Com a nova causa básica foram detectados também 19 acidentes de transporte $(27,9 \%)$, nove óbitos no grupo dos demais acidentes (13,2\%), quatro entre os suicídios $(5,9 \%)$ e 22 realocados nos homicídios (32,4\%) (Figura 1). Esses valores evidenciam uma proporção de resgate de informação bem mais elevada do que os descritos por

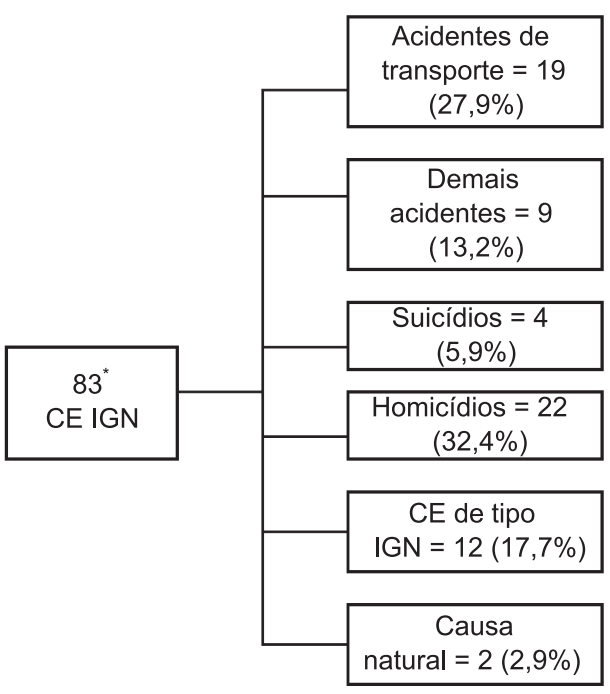

*Incluídas 15 perdas/Includes 15 losses

Figura 1 - Número e proporção (\%) de óbitos por causa externa totalmente mal definidos realocados segundo nova causa básica. Projeto SIM; 2000.

Figure 1 - Number and proportion (\%) of deaths due to completely ill defined external causes according to the new underlying cause. SIM Project; 2000.

Tabela 2 - Óbitos por causa externa de tipo ignorado (Y10 a Y34) e situação pós-investigação segundo tipo. Projeto SIM; 2000

Table 2 - Deaths due to external causes in the totally unknown group (Y10 to Y34) and situation after the investigation according to type. SIM Project; 2000

\begin{tabular}{lcccccccc}
\hline Causa original & \multicolumn{4}{c}{ Pós-investigação } & \multicolumn{3}{c}{ TOTAL } \\
\cline { 2 - 7 } & AT & D & S & H & IGN & CN & P & \\
\hline Causa externa totalmente ignorada (Y34) & 16 & 9 & 2 & 7 & 7 & 2 & 11 & 54 \\
Causa externa ignorada (Y10 a Y33) & 3 & - & 2 & 15 & 5 & - & 4 & 29 \\
- arma de fogo & - & - & $(1)$ & $(9)$ & $(3)$ & - & $(2)$ & $(15)$ \\
- instrumento cortante & - & - & - & $(4)$ & $(1)$ & - & $(2)$ & $(7)$ \\
- instrumento contundente & 3 & - & $(1)$ & $(1)$ & - & - & - & $(5)$ \\
- sufocação & - & - & - & $(1)$ & - & - & - & $(1)$ \\
- outro especificado & - & - & - & - & $(1)$ & - & - & $(1)$ \\
\hline TOTAL & 19 & 9 & 4 & 22 & 12 & 2 & 15 & 83 \\
\hline
\end{tabular}

AT $=$ acidentes de transporte; $\mathrm{D}=$ demais acidentes; $\mathrm{S}=$ Suicídios; $\mathrm{H}=$ Homicídios; $\mathrm{IGN}=$ ignorado; $\mathrm{CN}=$ Causa natural; $\mathrm{P}=$ perdas

AT $=$ transportation accidents; $\mathrm{D}=$ other accidents; $\mathrm{S}=$ Suicides; $\mathrm{H}=$ Homicides; $\mathrm{IGN}=$ Unknown: $\mathrm{CN}=\mathrm{Natural}$ causes: $\mathrm{P}$ $=$ losses 
Tabela 3 - Número e porcentagem de óbitos por acidentes de transporte terrestre de tipo ignorado (V89 - CID-10) segundo situação pós-investigação. Projeto SIM; 2000

Table 3 - Number and proportion (\%) of deaths due to motor-or non-motor-vehicle accidents of the unknown type (V89-ICD10) according to the situation after the investigation. SIM Project; 2000

\begin{tabular}{lcc}
\hline Situação pós investigação & No & $\%$ \\
\hline Perda & 1 & $*$ \\
Mantiveram-se como ignorados & 8 & 23,5 \\
Passaram a bem definidos & 26 & 76,5 \\
Pedestre & $(4)$ & $(11,8)$ \\
Ciclista & $(1)$ & $(2,9)$ \\
Motociclista & $(7)$ & $(20,6)$ \\
Ocupante veículo & $(9)$ & $(26,5)$ \\
Ocupante de caminhonete & $(1)$ & $(2,9)$ \\
Ocupante de veículo de transporte pesado & $(4)$ & 100,0 \\
\hline TOTAL & 35 & \\
\hline
\end{tabular}

\% calculadas com exclusão das perdas / \% calculated with exclusion of losses

Drumond et al. ${ }^{9}$, visto que esses autores não conseguiram esclarecer $66,4 \%$ dos casos analisados em São Paulo.

Esses resultados possibilitam mostrar que os Institutos de Medicina Legal, na grande maioria das vezes, dispõem de dados que permitem esclarecer melhor a causa de cada morte. O que geralmente ocorre é que essas informações não são transcritas nas declarações de óbito, fato que leva à presença de dados ainda incompletos e incorretos no Sistema de Informação de Mortalidade.

\section{Óbitos por causas externas com diagnóstico incompleto de nível I}

Compuseram esse grupo 73 óbitos distribuídos em 35 acidentes de transporte, 31 entre os "demais acidentes", dois suicídios e cinco homicídios (Tabela 1).

Das 35 mortes por acidentes de transporte nas quais a qualidade da vítima e as circunstâncias do acidente não eram conhecidas (casos codificados em V89 da CID-10) apenas um caso foi considerado "perdido", oito mantiveram-se na mesma situação e 26, correspondendo a $76,5 \%$, passaram a bem definidos (Tabela 3).

Quanto ao grupo dos "demais acidentes" (Tabela 4), verificou-se que 14 enquadraram-se na categoria de "exposição aci- dental a fatores não especificados" (X59 da CID-10), o que corresponde, simplesmente, à categoria "acidente".

Com relação aos 14 acidentes não especificados, a metodologia da investigação permitiu detalhar 13, dos quais 11 foram para acidentes de transporte terrestre, um para acidente causado por máquina e um para acidente causado por impacto de objeto. Dos que se tornaram acidente de transporte, a qualidade da vítima era pedestre em três casos, ciclista em um caso, motociclista em dois casos, ocupante de automóvel em quatro casos e ocupante de caminhonete em um caso. Comparando estes resultados com o trabalho realizado em São Paulo, relativo

Tabela 4 - Número de óbitos do grupo "demais acidentes com sub-tipo não especificado" segundo tipo, Projeto SIM; 2000.

Table 4-Number of deaths due to "other accidents of the unknown subtype" according to the type. SIM Project; 2000

\begin{tabular}{lr}
\hline TIPO & No \\
\hline Quedas & 2 \\
Afogamentos & 13 \\
Acidentes causados por fogo & 2 \\
Não especificados & 14 \\
\hline TOTAL & 31 \\
\hline
\end{tabular}


aos óbitos de $1996^{9}$, verificou-se que a quantidade de casos esclarecidos foi maior, visto que esses autores encontraram um resíduo de cerca de $20 \%$, e na atual pesquisa apenas um caso permaneceu como acidente de tipo ignorado. Quanto à nova causa básica, constatou-se que mais de $80 \%$ eram, na realidade, acidentes de transporte terrestre, valor que, naquele estudo, estava em torno de $30 \%$.

Quanto às quedas não especificadas, os dois casos puderam ser melhor detalhados, passando a uma queda bem definida e um acidente de transporte.

Para os dois casos inicialmente codificados como acidentes causados por fogo de tipo não especificado, verificou-se que em um foi mantido o diagnóstico e no outro a investigação levou à conclusão de homicídio.

A análise dos afogamentos e submersão acidentais (W65 a W74 da CID-10) mostrou aspectos interessantes. Dos 18 casos ocorridos dentre todas as causas externas, 13 $(72,2 \%)$ foram codificados como "afogamento sem outra especificação”. Estes, excluindo-se as duas perdas, transformaram-se em cinco afogamentos bem definidos, um homicídio por afogamento e cinco foram mantidos como afogamentos não especificados.

Quanto aos dois óbitos por suicídios, em um desses casos o laudo não foi localizado $\mathrm{e}$ no outro nada constava no documento que levasse a pensar em suicídio. Dessa forma, teoricamente o caso deveria passar a ser codificado como "evento de intenção ignorada”. A hipótese explicativa para que a informação sobre suicídio constasse apenas da DO original baseia-se no fato de que, ao preencher o documento, estando presente alguém da família, a informação deve ter sido dada por essa pessoa, não tendo sido a mesma transferida para o laudo.

Em relação aos homicídios, apenas cinco foram codificados como "agressão por meios não especificados (categoria Y09), sendo que, após a investigação, três tornaram-se bem definidos (X95; X99; Y00), um se manteve inalterado e, para um caso, não foi possível obter qualquer informação adicional (Perda). Pode-se referir, em razão dos achados, que o homicídio apresentou-se como um tipo de causa externa bem definido e no qual ocorreram poucas modificações após a investigação, já que a grande maioria se manteve como o mesmo tipo de homicídio; deve-se ressaltar, entretanto, que em um caso foi possível detalhar melhor o homicídio, codificando-o entre as intervenções legais, a partir da descrição das circunstâncias do evento prevista no novo modelo de DO implantada em 2000/2001 .

Na Figura 2 podem ser apreciadas de forma esquemática as mudanças ocorridas nos óbitos por causas externas com "diagnósticos incompletos". Assim, apesar de serem mantidas algumas freqüências, houve trocas importantes após a consulta às fontes mencionadas. Dos 38 óbitos que se tornaram acidentes de transporte bem definidos, 26 já o eram anteriormente, mas com diagnóstico incompleto, e 12 estavam classificados como "demais acidentes". Os cinco homicídios, reconhecidos como bem definidos após a investigação, foram decorrentes de duas mortes diagnosticadas como "demais acidentes" e três classificadas como homicídios, mas com diagnóstico incompleto.

\section{Óbitos por acidentes de transporte com diagnóstico incompleto de nível II}

A 10a Revisão da Classificação Internacional de Doenças, ao tratar dos acidentes de transporte, adota, como eixo classificatório, primeiramente a qualidade da vítima e em seguida a especificação do tipo de acidente, ou seja, considera importante serem analisados tanto o papel da vítima como as circunstâncias do acidente. O veículo ocupado pela pessoa traumatizada é codificado pelos dois primeiros caracteres, desde que se considere o mesmo como sendo o fator mais importante a ser identificado para fins de prevenção ${ }^{6}$. Assim, por exemplo, V01 a V09 identifica Pedestre traumatizado em acidentes de transporte, V10 a V19 Ciclista traumatizado...., enquanto o $3^{\circ}$ caracter, no caso de pedestres traumatizados, especifica o tipo de acidente: V01 - Pedestre traumatizado em colisão com veículo a pedal. 


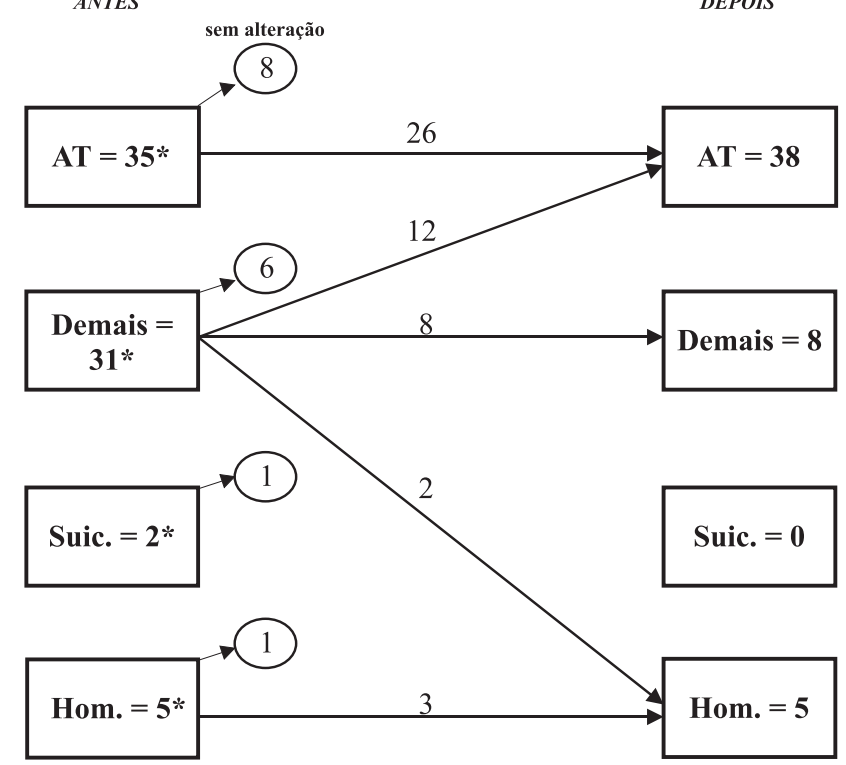

*Incluídas, respectivamente, 1, 3, 1 e 1 perdas/Included, respectively, 1, 3, 1 and 1 losses

Figura 2 - No de óbitos por causas externas com diagnóstico incompleto segundo tipo e situações antes e pós-investigação. Projeto SIM; 2000.

Figure 2 - Number of deaths due to external causes with incomplete diagnosis according to the new underlying cause. SIM Project; 2000

A distribuição dos óbitos por acidentes de transporte segundo os agrupamentos entre V01 e V99 pode ser apreciada na Tabela 5 .
Ao trabalhar apenas com os casos onde qualidade da vítima e as circunstâncias do acidente eram ignoradas, a análise foi feita somente com os 35 casos codificados em

Tabela 5 - Óbitos por acidentes de transporte segundo agrupamentos entre V01 e V99. Projeto SIM; 2000

Table 5 - Deaths due to motor-or non-motor-vehicle accidents according to groups (V01 to V99-ICD 10). SIM Project; 2000

\begin{tabular}{lrl}
\hline TIPO & No \\
\hline V01 a V09 & 19 (sendo 17 na categoria V09) $^{*}$ \\
V10 a V19 & 5 (sendo 3 na categoria V19) \\
V20 a V29 & 13 (sendo 7 na categoria V29) \\
V40 a V49 & 19 (sendo 17 na categoria V49) \\
V60 a V69 & 2 & \\
V80 a V89 & 41 (sendo 35 na categoria V89) \\
V98 e V99 & 1 & \\
\hline TOTAL & 100
\end{tabular}

"As categorias assinaladas, que totalizam 44 casos, correspondem a:

V09 Pedestre traumatizado em acidentes de transporte não especificados NE; V19 Ciclista traumatizado em acidentes de transporte NE; V29 Motociclista traumatizado em acidentes de transporte NE; V49 Ocupante de automóvel traumatizado em acidentes de transporte NE

* The categories checked, totaling 44 cases, refer to:

V09 Pedestrian hurt in non-specified transportation accidents (NE); V19 Bikers hurt in transportation accidents NE; V29 Motorcycle bikers hurt in transportation accidents NE; V49 Automobile occupants hurt in transportation accidents NE; 
V89 (acidentes de transporte terrestre, nos quais a qualidade da vítima e as circunstâncias do acidente não eram conhecidas) (casos vistos no item 3.2. redistribuídos segundo a Tabela 3 ).

Ao analisar também o detalhamento do tipo do acidente foi importante evidenciar a possibilidade de redistribuição das categorias V09, V19, V29, V49 e V69 (conforme já salientado, relativas ao conhecimento do tipo de vítima e desconhecimento do tipo de acidente), como está retratado na Tabela 6 .

Os achados evidenciam que nos 44 casos que englobaram as categorias referidas, em um não foi possível localizar o laudo. Dos 43 restantes, em 17 a informação tornou-se bem definida $(39,5 \%)$ para outros agrupamentos ou no mesmo agrupamento; em 26 $(60,5 \%)$ a informação da DO original não sofreu qualquer alteração, ou seja, os dados que constavam no IML eram os mesmos dados transcritos na DO.

A pesquisa permitiu ainda qualificar melhor as pessoas traumatizadas em acidentes de transporte. Mesmo para os casos não considerados categorias genéricas (V09, V19, V29 etc.) foram observadas algumas modificações após a investigação. Assim, os 17 pedestres reduziram-se a 13, os três ciclistas passaram a seis, os sete motociclistas tornaram-se nove e os 17 ocupantes de automó- vel transformaram-se em 12. Foram acrescidos um ocupante de caminhonete, um ocupante de transporte pesado, e um caso manteve-se entre os acidentes de tipo ignorado. É importante salientar que o conhecimento da qualidade da vítima permite programas específicos de prevenção. Assim, por exemplo, quanto aos motociclistas traumatizados em acidentes de transporte, que representavam 13\% na DO original (Tabela 5), com a metodologia da investigação, em suas várias etapas, passaram a vinte, acréscimo que correspondeu a 53,8\%.

Os resultados permitem de novo mostrar que os IML dispõem de informação bastante detalhada sobre os casos com necropsias, não transcrevendo os dados completos nas DO. Possibilitam ainda evidenciar que acréscimos significativos iriam ocorrer nas categorias bem definidas se as mal definidas ou incompletas pudessem ser realocadas.

\section{Análise de Ganhos}

Como decorrência dos resultados apresentados pode-se constatar que os objetivos propostos foram alcançados.

Embora ao longo do trabalho se tivesse podido constatar que algumas causas básicas originais que eram causas externas mal definidas ou com diagnósticos incompletos,

Tabela 6 - Número de óbitos por acidentes de transporte segundo qualidade da vítima e causa básica na DO original e pós-investigação. Projeto SIM; 2000

Table 6 - Number of deaths due to transportation accidents according to the victim and underlying cause in the original death certificate and after investigation. SIM Project; 2000

\begin{tabular}{|c|c|c|c|c|c|c|c|c|c|c|c|c|c|c|c|c|}
\hline \multirow{3}{*}{ DO ORIGINAL } & \multicolumn{14}{|c|}{ PÓS-INVESTIGAÇÃO } & \multirow{3}{*}{ Perdas } & \multirow{3}{*}{ Total } \\
\hline & \multicolumn{2}{|c|}{ Pedestre } & \multicolumn{2}{|c|}{ Ciclista } & \multicolumn{2}{|c|}{ Motociclista } & \multicolumn{2}{|c|}{$\begin{array}{c}\text { Ocup. } \\
\text { Automóvel }\end{array}$} & \multicolumn{2}{|c|}{$\begin{array}{c}\text { Ocup. } \\
\text { Caminhonete }\end{array}$} & \multicolumn{2}{|c|}{$\begin{array}{c}\text { Ocup. transp. } \\
\text { Pesado }\end{array}$} & \multicolumn{2}{|c|}{ Outros } & & \\
\hline & $\mathrm{BD}^{*}$ & V09 & $\mathrm{BD}$ & V19 & $\mathrm{BD}$ & V29 & $\mathrm{BD}$ & V49 & $\mathrm{BD}$ & V59 & $\mathrm{BD}$ & V69 & $\mathrm{BD}$ & V89 & & \\
\hline Pedestre V09 & 4 & 9 & 2 & 1 & - & - & - & 1 & - & - & - & - & - & - & - & 17 \\
\hline Ciclista V19 & - & - & 2 & 1 & - & - & - & - & - & - & - & - & - & - & - & 3 \\
\hline Motociclista V29 & - & - & - & - & 4 & 2 & - & - & - & - & - & - & - & - & 1 & 7 \\
\hline Ocupante automóvel V49 & - & - & - & - & 3 & - & 2 & 9 & - & 1 & - & 1 & - & 1 & - & 17 \\
\hline Ocupante caminhonete V59 & - & - & - & - & - & - & - & - & - & - & - & - & - & - & - & - \\
\hline Ocupante veículo pesado V69 & - & - & - & - & - & - & - & - & - & - & - & - & - & - & - & - \\
\hline TOTAL & 4 & 9 & 4 & 2 & 7 & 2 & 2 & 10 & - & 1 & - & 1 & - & 1 & 1 & 44 \\
\hline
\end{tabular}


ao serem investigadas mostraram-se de pior qualidade que as descritas na DO originalpelas razões já expostas - na análise dos ganhos esse aspecto não foi considerado. Esta decisão foi calcada no fato de que a metodologia da pesquisa estava dirigida a tentar melhorar a informação e, nos casos em que isso não era possível, ficava mantida a causa original.

Verificou-se que, embora tivesse havido número não desprezível de perdas $(13,5 \%$ para os dois subgrupos de causa externas), para os casos restantes pode-se dizer que em praticamente $80 \%$ foi conseguido algum tipo de ganho na nova causa básica, ou seja, a informação pôde ser melhorada em seus diferentes níveis (Tabela 7).
Naqueles casos onde nenhuma informação era conhecida, atingiu-se 82,4\% de melhor diagnóstico, sendo 60,3\% de diagnóstico bem definido. Mantiveram-se com o mesmo diagnóstico original $17,6 \%$ e, é importante notar, houve $18,1 \%$ de perdas. Nos casos em que se partiu de um diagnóstico incompleto nível I, as perdas foram menores $(8,2 \%)$, mas aqueles em que a causa básica inicial foi mantida elevaram-se para $23,9 \%$. Os ganhos foram obtidos em $58,2 \%$ dos casos () e em $17,9 \%$ ().

A análise dos ganhos do ponto de vista qualitativo (Tabela 8) permitiu conhecer a chamada "rede de migração" de causas, representada pelo número de óbitos em cada subgrupo do capítulo XX da CID-10, quantificado na DO original e na DO Nova.

Tabela 7 - Número e porcentagem de óbitos por causas externas segundo situação original e ganho de informação nas causas de morte. Projeto SIM; 2000

Table 7 - Number and proportion (\%) of deaths due to external causes according to original situation and gains in information on causes of deaths. SIM Project; 2000

\begin{tabular}{|c|c|c|c|c|c|c|}
\hline \multirow[t]{2}{*}{ Ganho } & \multicolumn{2}{|c|}{ Mal definidas } & \multicolumn{2}{|c|}{$\begin{array}{l}\text { Diagnóstico } \\
\text { incompleto }\end{array}$} & \multicolumn{2}{|c|}{ Total } \\
\hline & No & $\%^{*}$ & $\begin{array}{c}\text { Inco } \\
\mathrm{N}^{\circ}\end{array}$ & $\begin{array}{l}\text { ipleto } \\
\%^{*}\end{array}$ & No & $\%^{*}$ \\
\hline Causa inalterada & 12 & 17,6 & 16 & 23,9 & 28 & 20,7 \\
\hline Nova causa ainda totalmente incompleta & 4 & 5,9 & - & - & 4 & 3,0 \\
\hline Nova causa incompleta, melhor definida & 11 & 16,2 & 12 & 17,9 & 23 & 17,0 \\
\hline Nova causa bem definida & 41 & 60,3 & 39 & 58,2 & 80 & 59,3 \\
\hline Perdas & 15 & $18,1^{* *}$ & 6 & $8,2^{* *}$ & 21 & $13,5^{* *}$ \\
\hline$\overline{\text { Total }}$ & 83 & 100,0 & 73 & 100,0 & 156 & 100,0 \\
\hline
\end{tabular}

* Percentuais calculados sobre o total com exclusão das perdas/\% assessed based on total cases with exclusion of losses

** Calculadas sobre o total de casos/\% based on total cases

Tabela 8 - Número de óbitos segundo causa básica na DO original e pós-investigação. Projeto SIM; 2000. Table 8 - Number of deaths according to underlying cause in the original death certificate and pos-investigation. SIM Project; 2000

\begin{tabular}{lccccccccc}
\hline DO original & \multicolumn{8}{c}{ Pós-investigação } & \multicolumn{1}{c}{} \\
\cline { 2 - 8 } & $\begin{array}{l}\text { Acidentes } \\
\text { de transp. }\end{array}$ & Demais & $\begin{array}{c}\text { Suicí- } \\
\text { dios }\end{array}$ & $\begin{array}{c}\text { Homi- } \\
\text { cídios }\end{array}$ & Ign. & Sub-total & $\begin{array}{c}\text { Causas } \\
\text { naturais }\end{array}$ & Perdas & TOTAL \\
\hline Causas Externas & 65 & 24 & 4 & 27 & 13 & 133 & 2 & 21 & 156 \\
Acidentes de transporte & 34 & - & - & - & - & 34 & - & 1 & 35 \\
Demais aci-dentes & 12 & 15 & - & 1 & - & 28 & - & 3 & 31 \\
Suicídios & - & - & - & - & 1 & 1 & - & 1 & 2 \\
Homicídios & - & - & - & 4 & - & 4 & - & 1 & 5 \\
Ignorado & 19 & 9 & 4 & 22 & 12 & 66 & 2 & 15 & 83 \\
\hline Causas naturais & 2 & 10 & - & 1 & 2 & 15 & - & - & 15 \\
\hline TOTAL & 67 & 34 & 4 & 28 & 15 & 148 & 2 & 21 & 171 \\
\hline
\end{tabular}


Foi possível verificar que a pesquisa trouxe mudança significativa no panorama epidemiológico com relação às causas externas (Tabela 8).

Chama a atenção o fato de que, além do remanejamento entre os tipos de causas externas e dos dois casos que se tornaram causas naturais, já referidos, 15 mortes originalmente classificadas como decorrentes de causas naturais ${ }^{11}$ foram rediagnosticadas como causas externas (onze casos foram selecionados como mal definidos, na DO original, e quatro eram diagnósticos incompletos).

\section{Considerações Finais}

Embora se reconheça que, do ponto de vista da qualidade, as mortes decorrentes de acidentes e violências sejam melhor infor- madas na DO do que as causas naturais, há ainda muito a ser feito. A presente pesquisa mostrou que mudanças apreciáveis aconteceram na distribuição dos óbitos segundo o tipo de causa externa (Tabela 9), permitindo que houvesse um aumento significativo nos homicídios (razão igual a 5,6) e nos suicídios, que passaram a ter o dobro do número de casos. Os acidentes de transporte mostraram-se com $90 \%$ a mais de óbitos após a investigação, caindo sensivelmente a quantidade de mortes por causas externas do tipo ignorado.

É, portanto, indiscutível que, enquanto não se atinge um patamar ideal quanto ao preenchimento adequado das DO pelos legistas, a mera adoção desta metodologia simples, acessível e exeqüível - possibilita o desejado aprimoramento das estatísticas de mortalidade por causas externas.

Tabela 9 - Número de óbitos por causa externas segundo tipo, nas DO original e DO nova, e razão entre os mesmos. Projeto SIM, 2000.

Table 9 - Number of deaths due to external causes according to type, in the original and new death certificates, and ratio between them. SIM Project, 2000.

\begin{tabular}{lccc}
\hline Tipo & DO original (1) & DO Nova (2) & Razão (2)/(1) \\
\hline Acidente de transporte & 35 & 67 & 1,9 \\
Demais acidentes & 31 & 34 & 1,1 \\
Suicídio & 2 & 4 & 2,0 \\
Homicídio & 5 & 28 & 5,6 \\
Ignorados & 83 & 15 & 0,2 \\
\hline TOTAL & 156 & $148^{*}$ & \\
\hline
\end{tabular}

*Não incluídos as perdas e casos diagnosticados como mortes naturais

" Does not include losses and deaths due to natural causes

\section{Referências}

1. Mello Jorge, MHP, Gawryszewski VP e Latorre MRDO. Análise dos dados de mortalidade. IN Acidentes e violência no Brasil. Rev Saúde Pública 1997; 31 (Suppl 4): 5-25.

2. Mello Jorge MHP, Gotlieb SLD, Laurenti R. Situação de Saúde no Brasil: análise do período 1996 a 1999. Brasília (DF); 2002. OPS/OMS.
3. Yunes J, RAJS D. Tendencia de la mortalidad por causas violentas en la poblacion general y entre los adolescentes y jóvenes de la Región de las Americas. Cad Saúde Pública 1994; 10: 88-125.

4. Minayo MCS. A violência social sob a perspectiva da saúde pública. IN O impacto da violência social sobre a saúde. Cad Saúde Pública 1994; 10 Suppl: 7-18. 
5. Camargo JMB. Mortalidade por causas externas no Estado de São Paulo e suas regiões [Tese de Doutorado]. São Paulo: Faculdade de Saúde Pública da USP; 2002.

6. Organização Mundial de Saúde. Classificação estatística internacional de doenças e problemas relacionados à saúde, 10a Revisão (CID-10). São

Paulo: Centro Colaborador da OMS para a classificação de doenças em português; 1995, v.1.

7. Laurenti R, Cascão AM, Mello AP. The information about circunstances of external causes in the death certificates. Meeting of Heads of WHO Collaborating Centers for de Classification Diseases. Rio de Janeiro; 2000. (WHO, GPE, ICD/00,59)

8. Mello Jorge MHP. Situação atual das estatísticas oficiais relativas à mortalidade por causas externas. Rev Saúde Pública 1990; 24(3): 217-23.
9. Drumond Jr M et al. Avaliação da qualidade das informações de mortalidade por acidentes não especificados e eventos com intenção indeterminada. Rev Saúde Pública 1999; 33(3): 273-80.

10. Ministério da Saúde. Estatísticas de Mortalidade, Brasília (DF): FNA/MS; 1994.

11. Mello Jorge MHP, Gotlieb SLD, Laurenti R. O Sistema de Informação de Mortalidade: problemas e propostas para o seu enfrentamento. I Causas naturais. Rev Bras Epidemiol 2002; 5(2): 197-211.

12. Laurenti R, Mello Jorge MHP, 1996. $O$ atestado de óbito. São Paulo: Centro da OMS para a classificação de doenças em português; 1986 (Série Divulgação n.o 1).

13. Mello Jorge MHP, Koisumi MS. Acidentes de trânsito no Brasil: breve análise de suas fontes de dados. Revista da ABRAMET 2001; 38: 49-57.

Recebido em 22/03/02; aprovado em 05/09/02 